\title{
Legal Supervision Mechanism of Recommendation Algorithm Based on Intelligent Data Recognition
}

\author{
Siqi Zhong and Weier Zhang $(\mathbb{D}$ \\ School of International Business, Zhejiang Yuexiu University, Zhejiang 312000, China \\ Correspondence should be addressed to Weier Zhang; zhangweier@zyufl.edu.cn
}

Received 9 January 2022; Revised 7 February 2022; Accepted 8 February 2022; Published 24 February 2022

Academic Editor: Xin Ning

Copyright ( $) 2022$ Siqi Zhong and Weier Zhang. This is an open access article distributed under the Creative Commons Attribution License, which permits unrestricted use, distribution, and reproduction in any medium, provided the original work is properly cited.

\begin{abstract}
While considering the broad development prospects of intelligent investment advisers in the future, we must also be aware of the legal supervision issues that intelligent investment advisers bring with them. Simultaneously, big data, artificial intelligence, blockchain, and other technologies have advanced at a breakneck pace during this time. Many new technologies have been used in the financial sector. In the financial field, there is a trend toward gradual integration of finance and technology. The transformation of finance from concept to actual service has been realized thanks to emerging technologies. This combination of finance and technology is known as "financial technology." The legal supervision mechanism of a recommendation algorithm based on intelligent data recognition is investigated in this paper. On the one hand, it raises the bar for legal knowledge required by the legal supervision mechanism of recommendation algorithm, but on the other hand, it may reduce service efficiency and quality. As a result, using the understanding method of users' consulting intention while taking into account users' legal knowledge level, social attributes, and emotional status, it is a feasible and effective way to provide personalized and diverse legal consulting services to the general public.
\end{abstract}

\section{Introduction}

In recent years, with the continuous growth of the scale and maturity of the financial market, there is an urgent demand for financial innovation in the financial market. At the same time, big data, artificial intelligence, blockchain, and other technologies have also achieved leapfrog development during this period. Many emerging technologies have been applied to financial business. There is a trend of gradual integration of finance and technology in the financial field. Emerging technologies have realized the transformation from concept to actual service for finance. This combination of finance and technology is called "financial technology" [1]. While considering the broad development prospects of intelligent investment advisers in the future, we must also be aware of the legal supervision issues that intelligent investment advisers bring with them. After applying automation technologies such as algorithm and artificial intelligence to the field of legal supervision mechanism, algorithm recommended legal supervision mechanism is an unprecedented legal supervision mechanism [2]. The benefits of algorithm distribution include improving the efficiency of legal supervision mechanisms, alleviating information overload crises, and supply and demand crises, among others. However, as the information dissemination market has become more competitive, the platform's utilization goal has gradually shifted from meeting users' personalized needs to maximizing traffic. The recommendation algorithm has gradually evolved into a tool for the platform to increase market share, resulting in a misalignment of technical and value rationality: its widespread use has an impact on the spread of mainstream ideology. It has an impact on the information environment and the order in which information is disseminated in cyberspace, as well as changing users' information acquisition and reading habits and posing ideological security risks [3]. With the application of algorithm push in the field of legal supervision mechanism, new legal problems appear, and the traditional legal regulation is facing severe challenges. It is inevitable to seriously treat 
and deeply study the legal regulation of algorithm recommendation legal supervision mechanism $[4,5]$.

In recent years, information technology, such as blockchain and smart contract, with its advantages of low cost, high efficiency, and transparency, has been deeply integrated with traditional industries represented by finance, resulting in new industries and new formats, thus continuously promoting economic development $[6,7]$. However, there are many problems in this process, so it is necessary to take the law as the criterion, design the bottom line for it, and establish regulatory rules. Intelligent data recognition is mainly divided into two stages, the construction of classification learning model and the use of classification model. The core content of model building stage is classification modeling algorithm, which builds a classification function or model from data set for each category. Then, use the established classification model to classify and predict the data of unknown categories. How intelligent data identification identifies and classifies sensitive data, many conflicts have been reflected in previous studies. Some scholars have suggested that the legal supervision mechanism of recommendation algorithm should reflect the social values at that time, and the criteria for judging sensitive data are closely related to the social values $[8,9]$.

With the rapid development of intelligent data recognition technology, people can easily obtain a large amount of data, but it is difficult to obtain the hidden knowledge and internal relationship behind massive data by using traditional data processing and statistical analysis tools. The urgent need for technologies that can automatically and intelligently transform data into useful knowledge has promoted the rapid development of intelligent data recognition $[10,11]$. How to extract accurate, understandable, and usable knowledge and information from massive data has become one of the hot issues in the field of intelligent data recognition $[12,13]$. There is no effective legal service classification system from the perspective of the recommendation algorithm's legal supervision mechanism. On the one hand, it raises the bar for legal knowledge of the recommendation algorithm's legal supervision mechanism, while on the other hand, it may reduce service efficiency and quality [14]. As a result, it is a feasible and effective way to provide the public with personalized and diverse legal consulting services, as well as to develop a question-and-answer and intelligent dialogue engine with intelligent interaction capabilities for consultation, in the field of recommendation algorithm legal supervision mechanism.

\section{Related Work}

According to the literature [15], the legal supervision mechanism of the recommendation algorithm is part of the supervision mechanism. This viewpoint is supported by both America's Guide to Smart Investment Supervision and China's Opinion on Asset Management. And, after looking into the actual operation of smart investment, we discovered that the profit generated through investment advice corresponds to the definition of a security investment consulting organization under Chinese law. The concept is defined with reference [16] to the following factors, which is the theoretical and technical basis, using big data analysis and in accordance with the actual situation of the host country and region. It is primarily based on Markowitz's modern portfolio theory, the CAPM asset pricing model, behavioral finance, and other financial fundamental theories and models, as well as emerging technologies like artificial intelligence, big data, and cloud computing. According to the literature [17], the legal supervision mechanism of recommendation algorithms performs both investment consulting and asset management functions, the latter of which is the essence of smart investment. Due to legal differences between China and other countries, different perspectives on the essence of smart investment exist. According to the literature [18], the legal supervision mechanism of recommendation algorithms is inextricably linked to discretionary power, and because "asset management service" is an essential function of the legal supervision mechanism of recommendation algorithms, the fundamental sign for determining the true meaning of the legal supervision mechanism of recommendation algorithms is whether or not to adopt discretionary power. However, some academics disagree, believing that the legal oversight of recommendation algorithms should be limited to investment advice based on China's current artificial intelligence development level and the theoretical basis of securities. This view is more inclined to the definition of investment consultant in China's original legislation. Literature [19] through the method of big data analysis, the common operation process at present is to first understand the customer's situation through Internet questionnaires and Internet big data and other forms; judge the customer's risk preference, risk-taking ability, and other factors; realize the customer portrait; and then accurately provide personalized asset allocation services for customers according to this information. Literature [20] research shows that the legal supervision mechanism of recommendation algorithm faces double risks in the financial market: one is the existing liquidity risk and compliance risk in the market, and the other is the risk of personal information leakage and algorithm model defects caused by the close combination with Internet companies, and the latter has a greater impact. Literature [21] proposes whether the business type of the legal supervision mechanism of the recommendation algorithm only includes investment suggestions, or whether it also includes asset management services such as transaction execution and asset dynamic rebalancing. Therefore, from the perspective of what should be and the development trend of intelligent investment advisers in the future, China's intelligent investment advisers can be defined as providing customers with personalized investment advice services matching their portraits based on modern financial theories and models and relying on emerging technologies dominated by artificial intelligence asset management services. After combing the risk sources of the legal supervision mechanism of the recommendation algorithm through the big data analysis method, literature [22] found that the risks in traditional financial activities still continue to the intelligent investment adviser, and the legal supervision mechanism of the recommendation algorithm will even amplify the traditional risks, resulting in more serious consequences. Moreover, as the service basis of the legal supervision 
mechanism of recommendation algorithm, algorithm technology also brings challenges to intelligent investment advisory services. Literature [23] research shows that the basic basis for the survival of the legal supervision mechanism of recommendation algorithm is to realize the "preference feature" matching of news content based on the simple logic of "birds of a feather flock together and people flock together." Literature [24] proposed that the high risks of the legal supervision mechanism of the recommendation algorithm include the traditional risks of the financial market and the special risks brought by it as an auxiliary tool for financial activities, including technical risk, operational risk, agency risk, and information risk.

This paper investigates the legal supervision mechanism of recommendation algorithms using intelligent data recognition, as there is no direct legal regulation on algorithm supervision in China. China has chosen the legal regulation path of result supervision in response to the algorithm's negative legal consequences. Specifically, the algorithm's negative legal consequences are discovered through postevent content review, and the legal responsibility for these negative consequences is then assigned to the algorithm's developers or users' network platforms. The corresponding interest bias mode is established by calculating and analyzing the data and behavior of network users, and then, the text features of news content are calculated using word segmentation technology, resulting in a highly connected recommendation list and recommended content after the matching between "preferences" and "features" and personalized, targeted, and accurate distribution.

\section{Principle and Model of Intelligent Data Recognition}

Intelligent data recognition is the source of knowledge formation. It can be either structured, such as the data stored in the two-dimensional table of the database, or semistructured, such as office documents, text, reports, graphics and images, video and audio, or heterogeneous data from different networks. Intelligent data recognition has a much smaller sample size than most ethnic groups. However, the process of intelligent data recognition involves extracting information from a large number of incomplete, different, and random sample data, which may result in discrimination if only a small type of data can be selected. Sandbox aids in expanding the scope of legal supervision mechanism services of recommendation algorithms recommend algorithms and improve opportunities for legal supervision mechanism services, resulting in improved legal supervision mechanism results and ensuring that appropriate legal supervision mechanism protection measures are built in before legal supervision enters the mass market. The current measurement of the degree of the legal supervision mechanism in the research on the intelligent data recognition algorithm of the recommendation algorithm only considers the characteristics of the legal supervision mechanism, that is, using the definition of entropy in information theory to quantify the attribute legal supervision mechanism and ignoring the problem of individual privacy preference.
Therefore, the next step will be based on the algorithm. Consider training and identifying the data with personal privacy preferences, and continue to optimize the algorithm. Therefore, the sandbox model can bring great potential value to the recommendation algorithm legal supervision mechanism, innovative enterprises, regulators, and legal supervision market. FCA divides the whole process of supervision sandbox into seven steps and specifies each step, as shown in Figure 1.

Up to now, four batches of innovative projects have been tested for about four years since the release of the sandbox plan, and the operation experience of supervising the sandbox has gradually matured in this process. Among them, there are some test items with intelligent investment characteristics in each batch that apply for entering the sandbox and have successfully obtained the permission of FCA. The basic principle is to solve the problem of legal supervision mechanism by recommending algorithm. The deviation caused by the recommendation algorithm leads to different degrees of problems in legal supervision. Therefore, in order to reduce the above recognition problems caused by the recognition scene, the feature scale is designed with the red scale as the target recognition object, and the standard is attached as a reference to realize the control of legal supervision. The designer of intelligent data identification algorithm should record its data collection mode, source, content, and processing process for inquiry and be obliged to explain the relevant principles of intelligent data identification algorithm technology. At the same time, the designer should be held accountable for the discriminatory algorithm designed out of malicious intent. The main idea of this method is to calculate the legal supervision mechanism through the proportional conversion between the statistics of the characteristic legal supervision mechanism and the known recommendation algorithm and then complete the automatic identification of the legal supervision mechanism. The processing method is triggered by the alarm function of the client; that is, when the legal supervision mechanism changes, the alarm callback function is triggered, and real-time snapshot analysis is carried out. The method can be roughly divided into five steps, as shown in Figure 2.

This method is based on intelligent data recognition technology and combined with recommendation algorithm to realize the identification of legal supervision mechanism. Compared with the designers of intelligent data identification algorithms, the users of intelligent data identification algorithms act directly on the algorithm recipients through algorithms and are the information trustees of algorithm service recipients such as customers and consumers. Therefore, the algorithm users need to fulfill the obligation of loyalty and shall not obtain relevant personal information by deception, abuse data information, and store data. Users have the duty of care to prevent information disclosure and improper use. In the whole process of the legal supervision mechanism of intelligent data recognition algorithm recommendation, the algorithm creator provides the algorithm platform, the algorithm application subject provides the news content, and the algorithm recommendation news receiver provides the digitized individual attribute. The three 


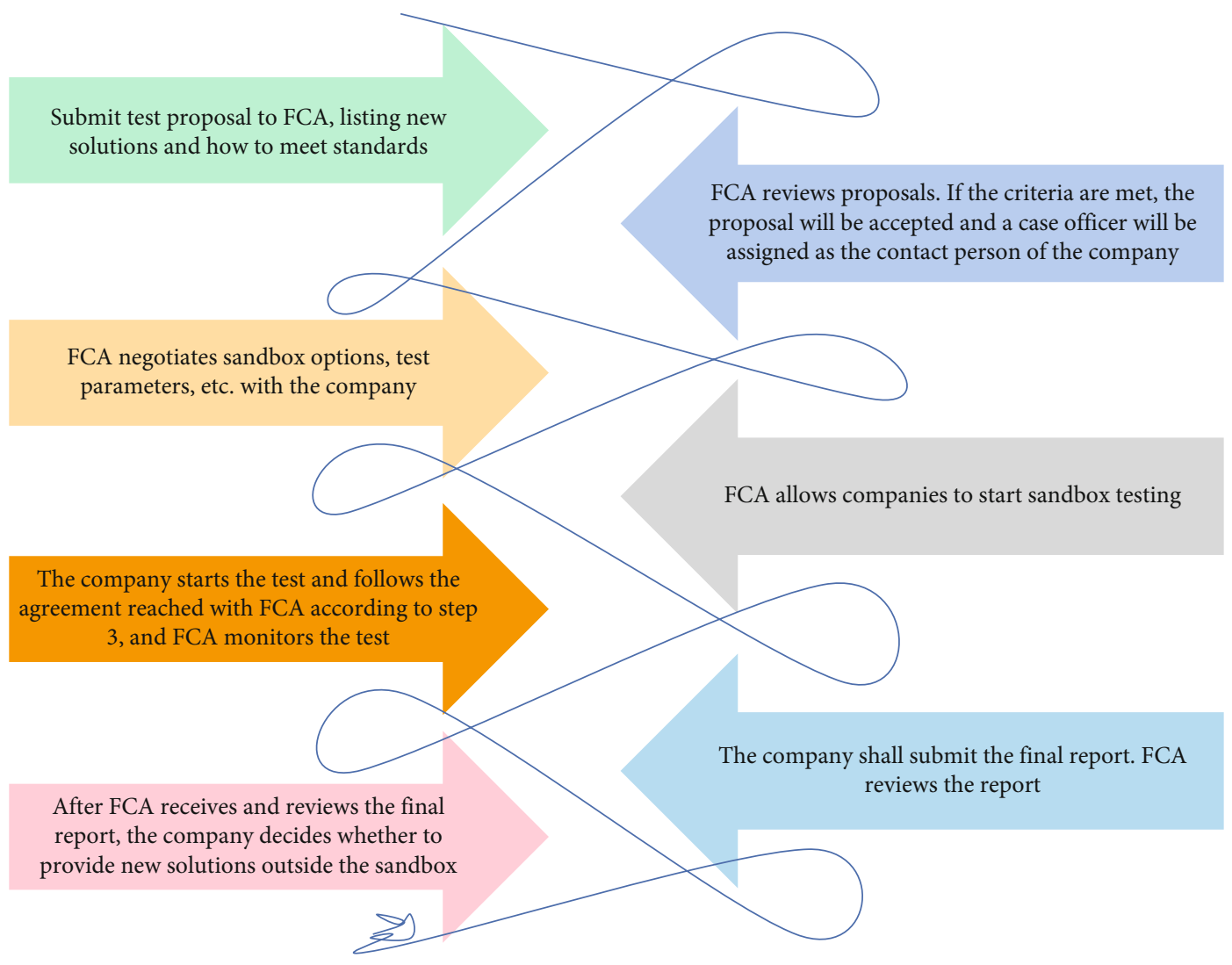

FIGURE 1: Sandbox process of legal supervision mechanism.

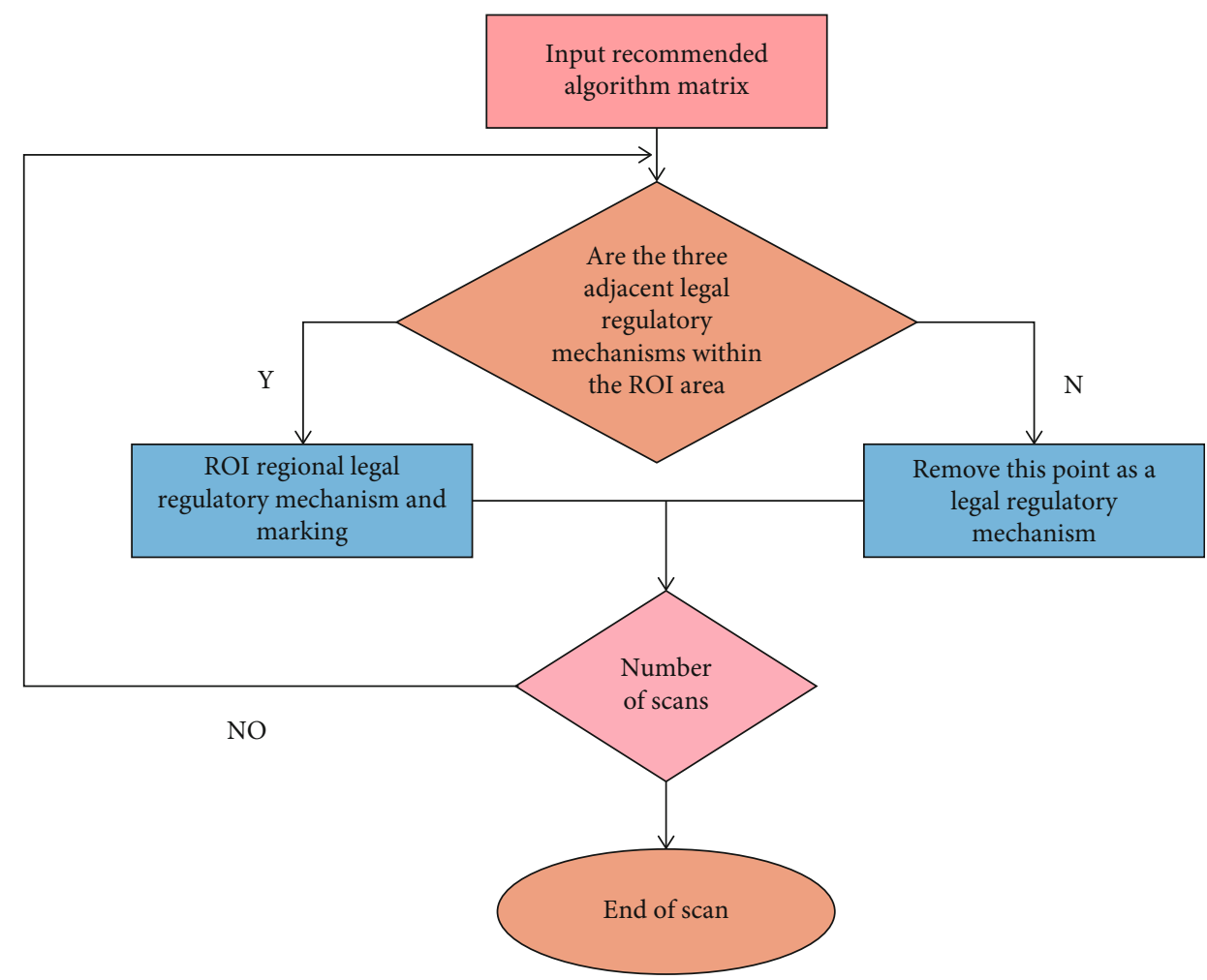

FIGURE 2: Recommended algorithm legal supervision mechanism processing. 


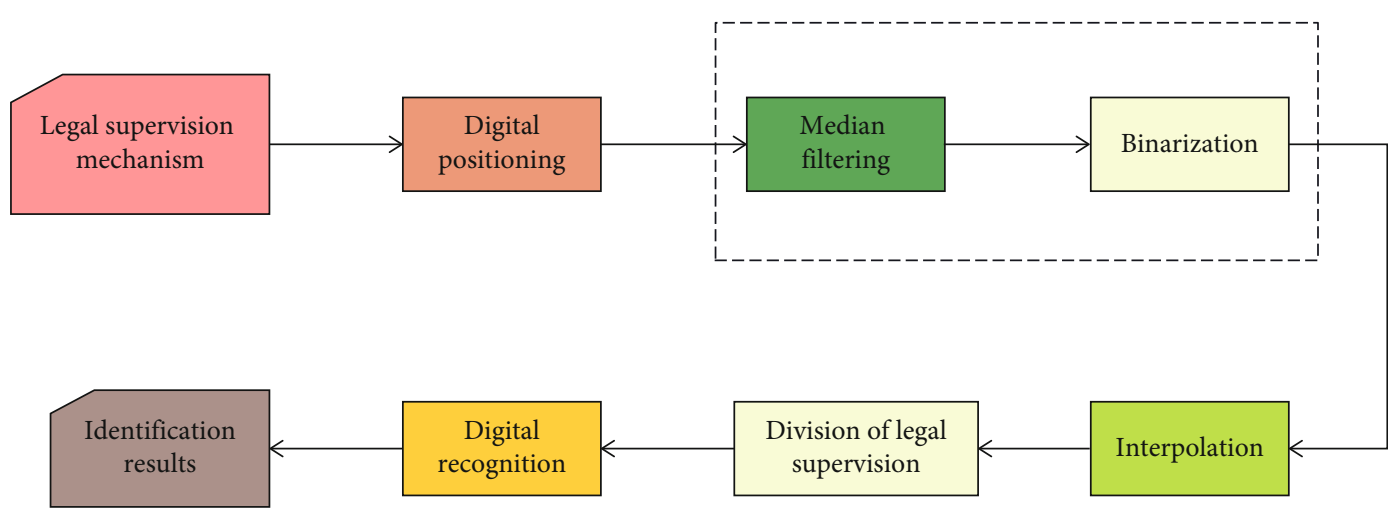

FIgURE 3: Flow chart of intelligent data identification.

together form an interconnected and mutually accessible system based on the algorithm rules. The basic idea of this method is as follows: firstly, locate the area of intelligent data to be identified in order to correctly locate the digital area to be identified; median filter and binarization are used to extract interested binarization results from complex background. The interpolation module solves the problem of low resolution. The segmentation and recognition module can segment and recognize the number, respectively. This method can directly read the mark on the grain scale as the number of grain storage height in the granary. The overall process of intelligent data recognition is as follows, as shown in Figure 3.

Extracting valuable knowledge from a large amount of data, which combines traditional data analysis methods with complex algorithms for processing a large amount of data, can be widely used in product control, market analysis, decision support, scientific exploration, and other fields. The governance of discrimination based on intelligent data identification algorithm is difficult and heavy, and government intervention alone is far from enough. Only by the joint efforts of many subjects and the cogovernance of government supervision, industry self-discipline and personal supervision can the best effect be achieved. Relevant Internet industry associations should play their leading role and guide Internet enterprises to build equal, safe, and respectful algorithmic ethics to realize industry autonomy. The off-line nonlinear model $F()$ can not represent the current working conditions of industrial processes with time-varying characteristics. The input-output relationship of the industrial process model at time $\mathrm{mn}$ is expressed by the following formula.

$$
y_{m_{n}}=f^{\prime}\left(x_{m_{n}}\right), m_{n}=k+1, k+2, \cdots
$$

The process monitoring method based on PCA has been successfully applied in industrial processes with time-varying characteristics such as chemical industry and semiconductor manufacturing. The PCA model is constructed by using the training data of off-line model $f(\cdot)$ Divide the calibrated new sample $X_{K+1}$ into two parts

$$
\left\{\begin{array}{l}
x_{k+1}=\hat{x}_{k+1}+\tilde{x}_{k+1}, \\
\hat{x}_{k+1}=x_{k+1} \hat{P}_{k} \stackrel{\wedge}{P}_{k}^{T} \\
\tilde{x}_{k+1}=x_{k+1}\left(I-\hat{P}_{K} \stackrel{\wedge}{P}_{k}^{T}\right),
\end{array}\right.
$$

where $\hat{P}_{k}$ is the load matrix and $\hat{x}_{k+1}$ and $\tilde{x}_{k+1}$ are the projections of $x_{k+1}$ on the principal subspace and residual subspace of PCA model.

Calculate the SPE and Hotelling's T2 for the new sample:

$$
\begin{aligned}
& \left\{\begin{array}{l}
\hat{t}_{k+1}=x_{k+1} \hat{P}_{k}, \\
\hat{x}_{k+1}=\hat{t}_{k+1} \stackrel{\wedge}{P}_{k}^{T} \\
\tilde{x}_{k+1}=x_{k+1}-\hat{x}_{k+1}, \\
\mathrm{SPE}=\left\|\tilde{x}_{k+1}\right\|^{2}=\left\|x_{k+1}\left(I-\hat{P}_{K} \stackrel{\wedge}{P}_{K}^{t}\right)\right\|^{2}, \\
\hat{T^{2}=} x_{k+1} \hat{P}_{K} \hat{\Lambda}^{-1} \hat{P}_{k}^{T} x_{k+1}^{T}, \\
\hat{\Lambda}_{k}=\frac{\hat{T}_{k}^{T} \hat{T}_{k}}{k-1}=\operatorname{diag}\left\{\lambda_{1}, \lambda_{2}, \cdots, \lambda_{h}\right\},
\end{array}\right.
\end{aligned}
$$

where $\hat{\Lambda}_{k}$ is the eigenvector composed of the first $h$ eigenvalues and $\hat{T}_{k}$ is the score matrix. Generally, SPE is used to measure the projection of the new sample on the residual subspace, indicating the degree to which the new sample deviates from the model; T2 measures the change of the new sample in the principal component subspace, representing the degree of deviation of the new sample in the model. If SPE and T2 model update is not performed if the following conditions are met.

$$
\left\{\begin{array}{l}
\mathrm{SPE} £ \mathrm{SPE}_{\alpha_{\mathrm{pro}}} \\
T^{2} \leq T_{\alpha_{\mathrm{pro}}}^{2}
\end{array} .\right.
$$


In which, SPE $\alpha$ pro and T2 $\alpha$ pro represent the control limits of SPE and T2.

Compared with the modeling samples, the new samples collected in the industrial process usually have abrupt changes and slow changes. The ALD value between the new samples and the modeling samples is used to describe this change, which is defined as follows

$$
\delta_{k+1}=\min \left\|\sum_{l=1}^{k} \alpha_{l} x_{l}-x_{k+1}\right\|^{2} .
$$

Among them, $\delta_{K+1}$ can be obtained by solving in the original space or kernel space of the input sample be based on $\delta_{K+1}$ and the threshold $V$ set according to experience, judge whether to update the model, and then control the number of model updates: if $\delta_{K+1}$ is less than or equal to the set threshold $V$ and the model is not updated. Otherwise, it indicates that the new sample is relatively independent of the modeling sample, and the model is updated. When PEB meets the following conditions, the model is not updated.

$$
e_{m_{n}} \leq \operatorname{Rule}\left(\left\{\delta_{m_{n}}^{1}, \delta_{m_{n}}^{2}, \cdots\right\}\right)
$$

where $e_{m}$ represents different thresholds set according to prior knowledge, and rule $(\cdot)$ represents judgment rules set according to experience. The error EMN is calculated by the following formula:

$$
e_{m_{n}}=y_{m_{n}}-f\left(x_{m_{n}-1}\right), m_{n}=k+1, k+2, \cdots
$$

This method predefines multiple parameters according to the actual needs $\delta \mathrm{Mn}$ threshold, and corresponding rules identify the updated samples. Intelligent data recognition is an important task of data mining. It can be used to mine the models of important data classes and predict the future trend of data. Intelligent data recognition has been widely studied in the fields of network finance, machine learning, expert system, pattern recognition, artificial intelligence, and so on.

\section{Legal Supervision Path of Recommendation Algorithm}

4.1. Legal Supervision Mechanism of Recommendation Algorithm Based on Intelligent Data Identification. In recent years, the scope of content review on the adverse consequences caused by the legal supervision mechanism of recommendation algorithms in various countries has generally expanded. Taking the proliferation of fake news on social media as an example, countries have generally strengthened the content censorship of social media, not only strictly supervising the illegal content that infringes public interests such as horror, pornography, and hatred but also strengthening the supervision of the content that infringes private rights by defamation. Usually, the legal supervision mechanism process of recommendation algorithm can only obtain the true value corresponding to that moment after a period of intelligent data identification and measurement, and the lag time varies with different industrial processes. Aiming at the problems that the recommendation algorithm of intelligent data identification has little consideration for the classification of legal supervision mechanism and unreasonable noise addition, in order to avoid the problem of insufficient or excessive protection of legal supervision mechanism in data set, which leads to poor data utility, an adaptive privacy protection scheme of legal supervision mechanism for structured data set is proposed. That is to say, we first identify the measurement based on intelligent data and then update the model online according to the true values obtained by other means such as the legal supervision mechanism of recommendation algorithm, so as to serve the measurement of the legal supervision mechanism of recommendation algorithm at the next moment; that is, it is divided into two stages: online measurement and online update. China has always attached great importance to the supervision of legal supervision mechanism of recommendation algorithm. The Administrative Measures for Internet Information Services, implemented in 2000, as a basic regulation, stipulates eight contents that legal supervision mechanism providers are not allowed to make, copy, publish, or disseminate, covering illegal contents such as "opposing the basic principles defined in the Constitution" and "endangering national security" and has set a blanket clause "including other contents prohibited by laws and administrative regulations." In the past year or two, new regulations have been introduced intensively, further expanding the scope of content censorship, such as "insulting or slandering others, infringing on the legitimate rights and interests of others" and vague moral regulations "endangering social morality or national excellent cultural traditions," etc.

For the user consultation and service system of legal supervision mechanism of recommendation algorithm, the understanding and identification of user intention in the process of user consultation is the key step. User's intelligent data recognition session intention understanding is usually regarded as a semantic discourse classification problem; that is, the user's consultation content is automatically classified into predefined semantic categories. Accurate user intelligent data recognition session intention understanding can increase constraints to narrow the retrieval scope of subsequent content search, which is conducive to the retrieval and screening of bill provisions, laws and regulations, similar cases, etc. Different recommendation algorithms have different advantages and disadvantages. The media platform should combine a variety of algorithms to avoid or reduce the shortcomings of a certain algorithm, especially for vulgar content. In the early stage of the development of legal supervision mechanism, if the adverse legal consequences caused by the recommendation algorithm, such as the legal supervision mechanism consequences of copyright infringement caused by the recommendation algorithm of content distribution, the network platform shall bear the civil tort liability. General network platforms can claim exemption through the "safe haven" rule without providing content or performing the obligation of reasonable care, which has also been adopted by many countries in the world. 


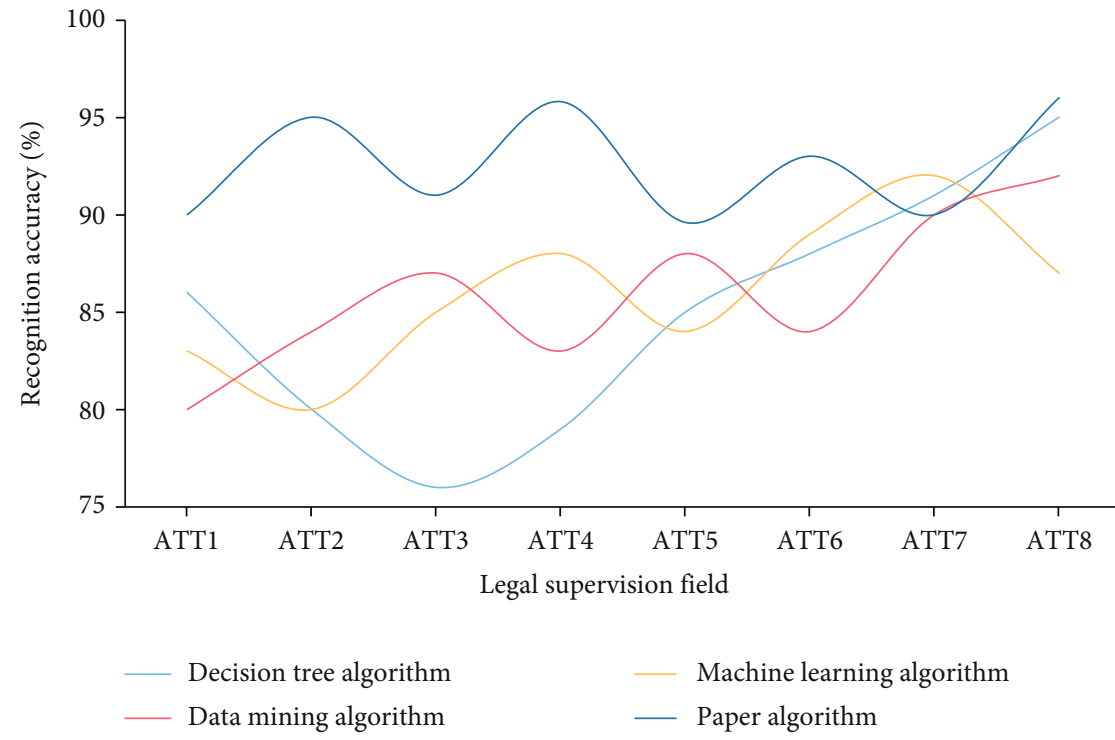

Figure 4: First comparison of the accuracy of different algorithms on legal supervision mechanism.

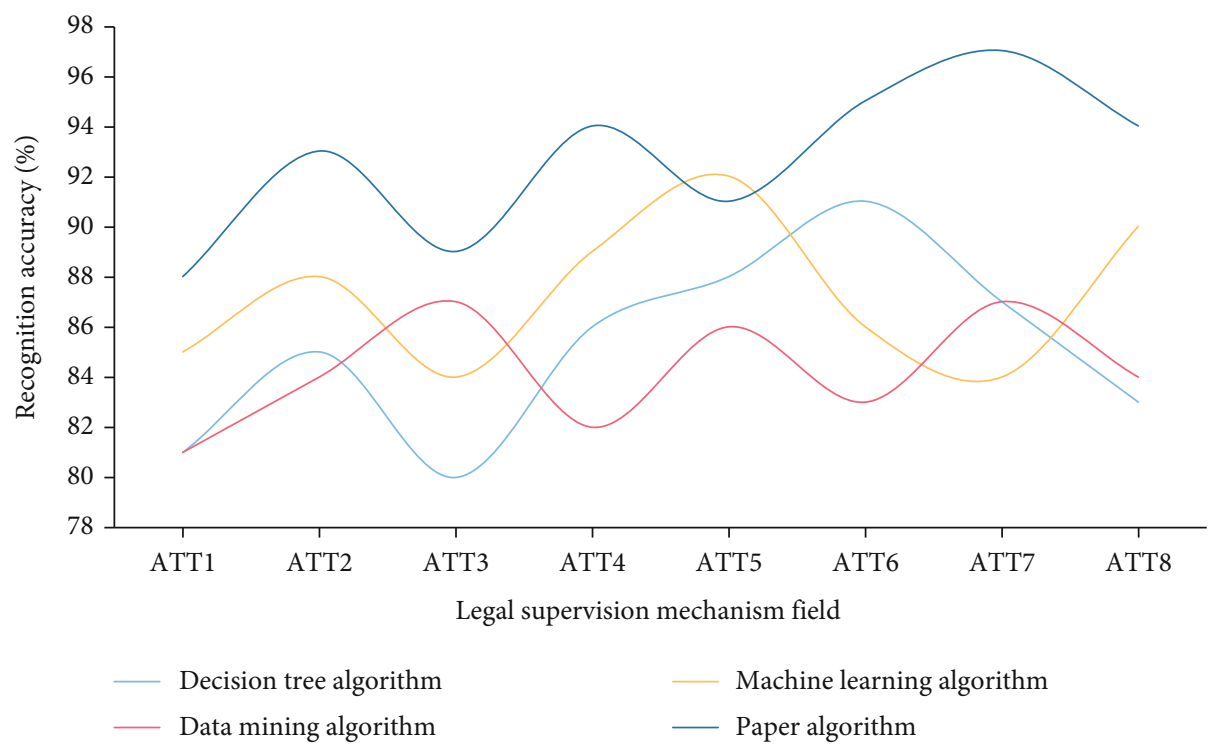

FIGURE 5: Second comparison of accuracy of different algorithms on legal supervision mechanism.

4.2. Experimental Results and Analysis. In order to more intuitively reflect the advantages of the algorithm proposed in this paper, the decision tree algorithm, machine learning algorithm, data mining algorithm, and the methods proposed in this algorithm are compared with this algorithm. By identifying the data of legal supervision mechanism in the same data set, three experiments are conducted in this experiment, and a comparative analysis is made from two aspects of efficiency and accuracy, as shown in Figures 4-6.

The accuracy comparison of different algorithms for sensitive attribute recognition is shown in the figure based on the experimental results. It can be seen that this algorithm's average recognition accuracy for the legal supervision mechanism in the data set is $95 \%$, which is higher than other algorithms' recognition accuracy. The decision tree algorithm can identify five sensitive attributes in the data set but not att2, att3, att5, or att6, and the recognition accuracy is low. There are drawbacks to using a data mining algorithm to identify sensitive attributes. Because the sensitive fields are predefined, only three predefined sensitive attributes can be identified for the experimental data set, and because the other sensitive fields do not match the predefined sensitive fields, they cannot be identified as sensitive attributes, and the identification accuracy is the lowest of all algorithms. The method of machine learning algorithm can identify five sensitive attributes, and its recognition accuracy is slightly higher than that of decision tree algorithm. Both data mining algorithm and decision tree algorithm can identify 9 sensitive attributes, and their average recognition accuracy is almost the same, which are $82.5 \%$ and $83.7 \%$, respectively. 


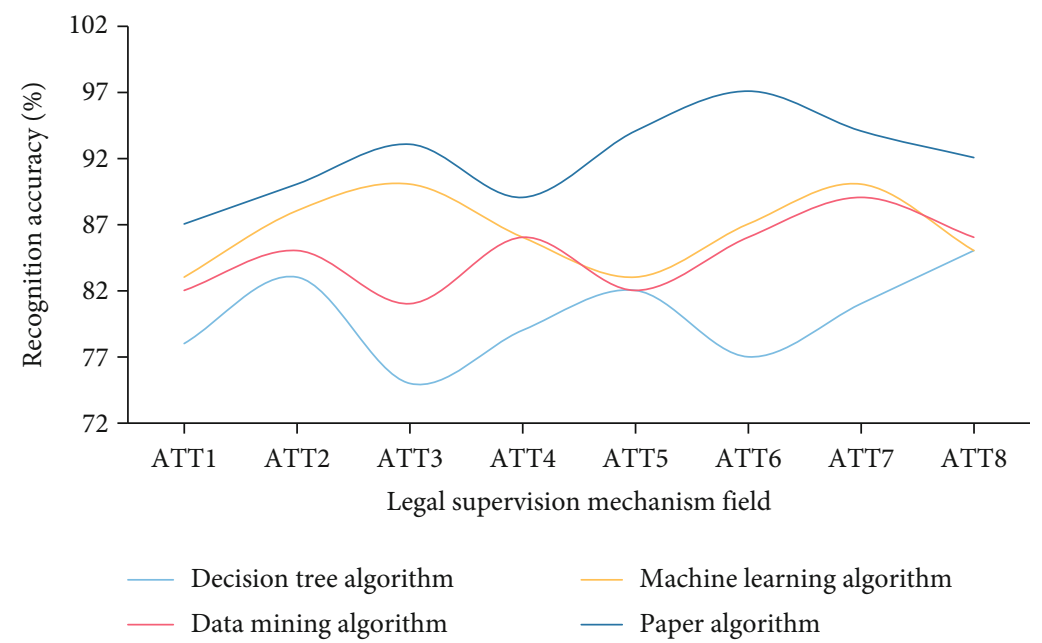

FIGURE 6: The third comparison of the accuracy of different algorithms on legal supervision mechanism.

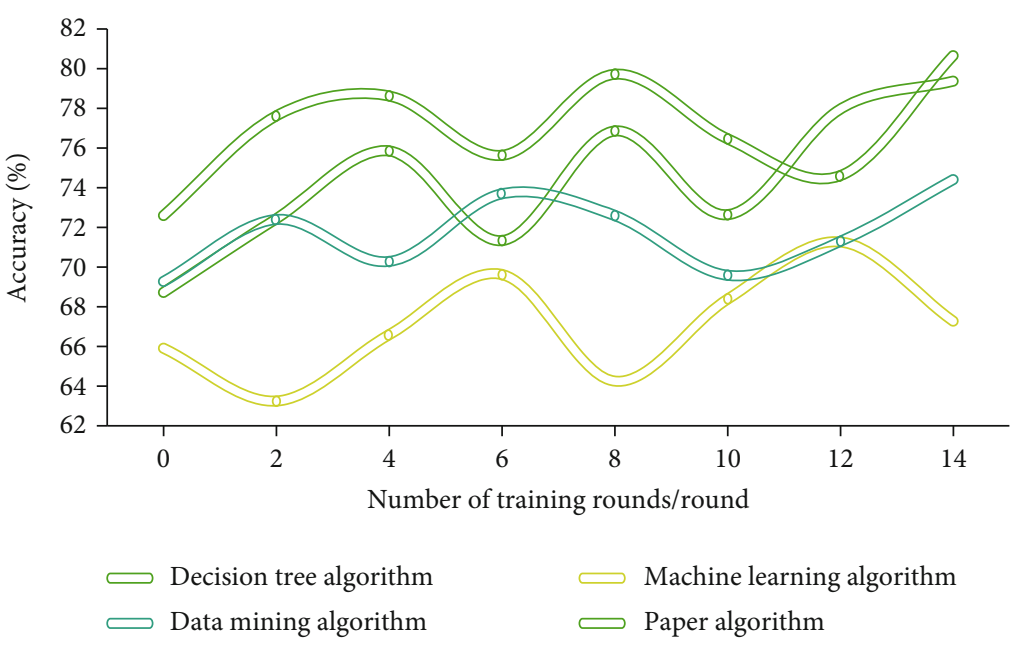

Figure 7: Impact of data set training rounds on the accuracy of legal supervision mechanism.

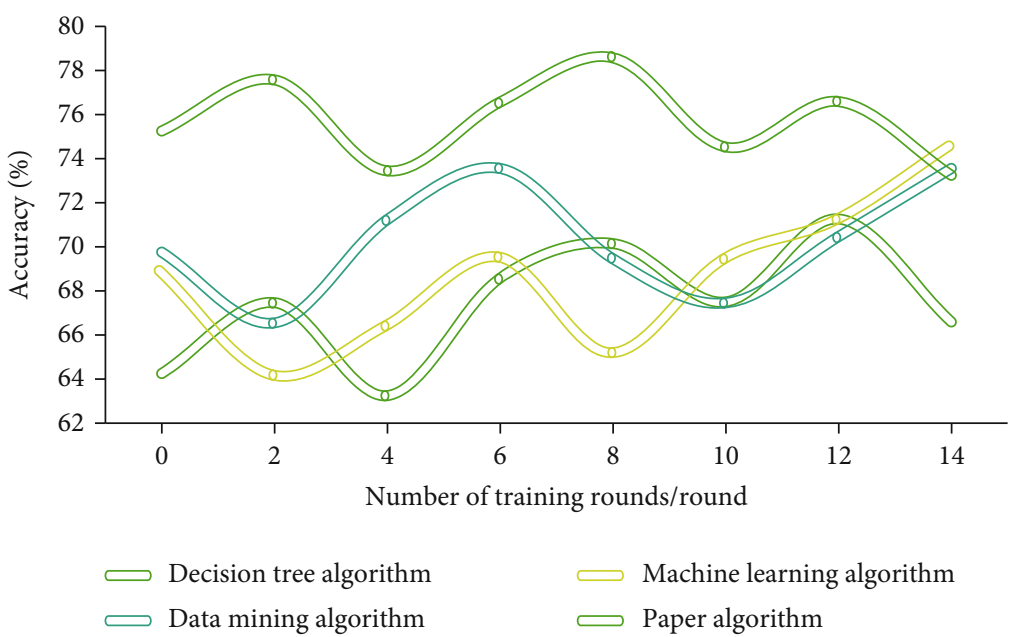

FIGURE 8: Impact of training rounds of data set on the accuracy of legal supervision mechanism. 


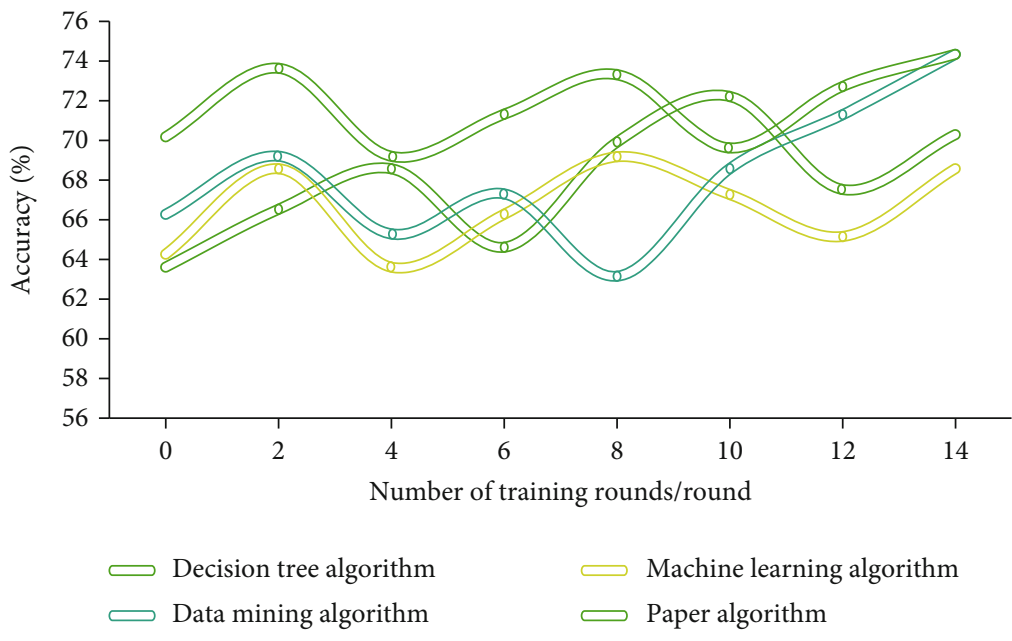

FIGURE 9: Impact of training rounds of data set on the accuracy of legal supervision mechanism.

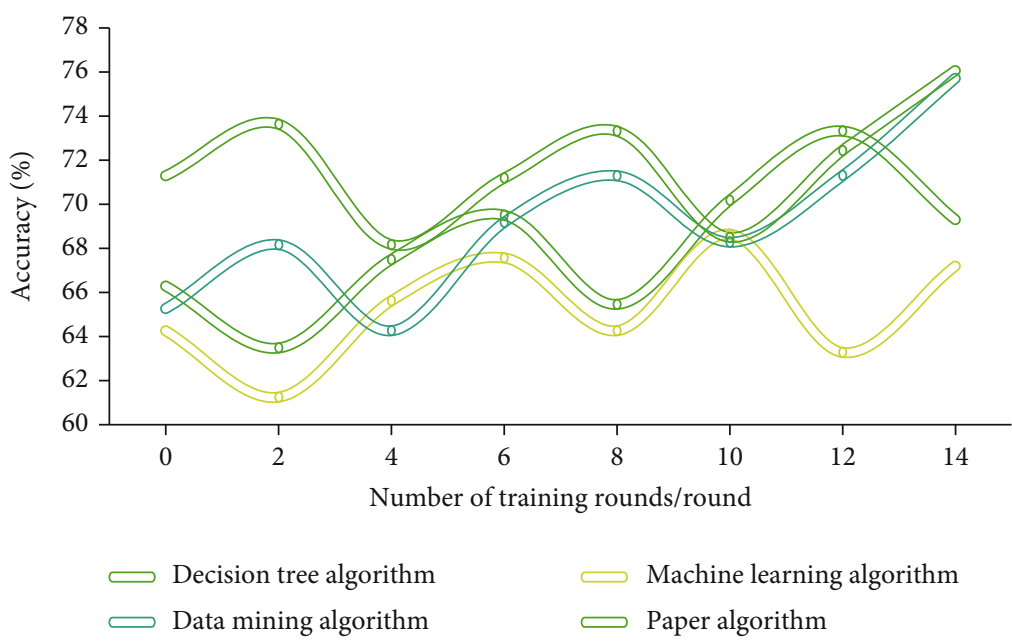

FIGURE 10: Impact of training rounds of data set on the accuracy of legal supervision mechanism.

The recommendation algorithm based on intelligent data recognition is also an influencing factor of word vector quality, and the number of training rounds of the regulatory mechanism training set by law is related to experiments and analysis in this section. The experiment is carried out by using decision tree algorithm, machine learning algorithm, data mining algorithm, and this algorithm. The dimension of word vector is 300 dimensions, the window size is 15 , the minimum word frequency is 5 , and the rest are the default parameters. The experiment only changed the number of training rounds, and four experiments were conducted, respectively. The experimental results are shown in Figures 7-10.

As can be seen in Figures 7-10, with the increase of the number of training rounds, the classification accuracy of the data set in the simple softmax regression model gradually increases and remains stable when reaching a certain number of training rounds. The number of training rounds of word vector training in the data set is about 5-10, considering the time consumption and classification effect of word vector training. The scope of content review of illegal net- work information dissemination is growing by the day, implying that legislators believe that developers and users of network platforms and other software can fully control the production and dissemination of information and have "all-round" ability and authority to review network information. A classification and classification method of legal supervision mechanisms for structured data sets is proposed under the framework of futurism to address the problems of different categories of legal supervision mechanisms and diversified legal supervision mechanisms involved in the recommendation algorithm, in order to prevent too much privacy data loss and to comprehensively consider the mutual information correlation coefficient and correlation relationship between attributes.

\section{Conclusions}

“The legal system's value and significance lie in standardizing and pursuing every detail of technically manageable risks and disasters, even if the possibility or scope of influence is small." The main determinant of fuzzy rules for intelligent 
data identification is domain experts' experience. In practice, it is necessary to extract the application objects of a specific recommendation algorithm and legal supervision mechanism, as well as provide a customizable human-computer interaction interface. This paper proposes an intelligent data identification algorithm in light of the fact that the existing data identification algorithm and privacy protection algorithm of legal supervision mechanisms cannot meet the diversified and numerous privacy protection requirements of data in the era of big data. To some extent, sensitive attributes must be classified and graded before being protected in order to prevent large data loss. This paper proposes a recommended algorithm for intelligent data identification, as well as a classification method for legal supervision mechanisms, based on the aforementioned problems. The restriction of personal data collection and use behavior, the establishment of mandatory algorithm disclosure obligations at various levels to increase algorithm transparency, and the creation of the right to interpret algorithms to provide relief to interested parties are all addressing the risks posed by the advancement of science and technology from different perspectives. If the network platform bears civil tort liability in the early stages of the development of legal supervision mechanism, such as the legal supervision mechanism consequences of copyright infringement caused by the recommendation algorithm of content distribution, the network platform will bear civil tort liability.

\section{Data Availability}

The data used to support the findings of this study are included within the article.

\section{Conflicts of Interest}

The authors do not have any possible conflicts of interest.

\section{References}

[1] T. C. Chang, E. A. Vanner, D. Fujino, S. Kelly, and R. K. Parrish, "Factors associated with laser trabeculoplasty response duration: analysis of a large clinical database (IRIS Registry)," Journal of Glaucoma, vol. 30, no. 10, pp. 902-910, 2021.

[2] J. Gao, Y. Liu, F. Yang, X. Chen, B. F. Cravatt, and C. Wang, "CIMAGE2.0: an expanded tool for quantitative analysis of activity-based protein profiling (ABPP) data," Journal of Proteome Research, vol. 20, no. 10, pp. 4893-4900, 2021.

[3] L. A. Adutwum, K. Joanna Koryo, and J. J. Harynuk, "Unique ion filter-a data reduction tool for chemometric analysis of raw comprehensive two-dimensional gas chromatography-mass spectrometry data," Journal of Separation Science, vol. 44, no. 14, pp. 2773-2784, 2021.

[4] J. Im, S. Sen, S. Lindsay, and P. Zhang, "Recognition tunneling of canonical and modified RNA nucleotides for their identification with the aid of machine learning," Acs Nano, vol. 12, no. 7, pp. 7067-7075, 2018.

[5] X. Li, R. Liu, H. Yan et al., "Effect of CYP2C9-VKORC1 interaction on warfarin stable dosage and its predictive algorithm," Journal of Clinical Pharmacology, vol. 55, no. 3, pp. 251-257, 2015.
[6] S. Ahmadian, M. Meghdadi, and M. Afsharchi, "A social recommendation method based on an adaptive neighbor selection mechanism," Information Processing \& Management, vol. 54, no. 4, pp. 707-725, 2018.

[7] C. H. Chen, Y. H. Chen, V. G. Diaz, and J. C. Lin, "An intelligent trading mechanism based on the group trading strategy portfolio to reduce massive loss by the grouping genetic algorithm," Electronic Commerce Research, vol. 10, pp. 1-40, 2021.

[8] P. Jiang, J. Winkley, C. Zhao, R. Munnoch, G. Min, and L. T. Yang, "An intelligent information forwarder for healthcare big data systems with distributed wearable sensors," IEEE Systems Journal, vol. 10, no. 3, pp. 1147-1159, 2016.

[9] M. L. Lee, T. W. Ling, and W. L. Low, "Intelliclean: a knowledge-based intelligent data cleaner," in In Proceedings of the sixth ACM SIGKDD international conference on Knowledge discovery and data mining, pp. 290-294, New York, 2000, August.

[10] A. Ashu, M. W. Hussain, D. Sinha Roy, and H. K. Reddy, "Intelligent data compression policy for Hadoop performance optimization," in International Conference on Soft Computing and Pattern Recognition, pp. 80-89, Springer, Cham, 2021.

[11] H. Salehi, S. Das, S. Biswas, and R. Burgueño, "Data mining methodology employing artificial intelligence and a probabilistic approach for energy-efficient structural health monitoring with noisy and delayed signals," Expert Systems with Applications, vol. 135, pp. 259-272, 2019.

[12] F. A. Khan, A. Khalid, M. Ali, M. Imran, M. Nawaz, and A. Rahman, "An intelligent data service framework for heterogeneous data sources," Journal of Grid Computing, vol. 17, no. 3, pp. 577-589, 2018.

[13] T. Ibrayev, U. Myrzakhan, O. Krestinskaya, A. Irmanova, and A. P. James, "On-chip face recognition system design with memristive hierarchical temporal memory," Journal of Intelligent \& Fuzzy Systems, vol. 34, no. 3, pp. 1393-1402, 2018.

[14] B. He and G. Li, "Intelligent self-adaptation data behavior control inspired by speech acts," ACM Transactions on Sensor Networks, vol. 13, no. 2, 2017.

[15] N. Lu, S. Wang, and H. Xiao, "An unsupervised intelligent fault diagnosis system based on feature transfer," Mathematical Problems in Engineering, vol. 2021, no. 4, Article ID 6686057, p. 12, 2021.

[16] J. B. Guerard Jr., S. T. Rachev, and B. P. Shao, "Efficient Global Portfolios: Big Data and Investment Universes," in Handbook of Applied Investment Research, pp. 357-367, World Scientific Publishing, 2020.

[17] S. Schuman and A. Lin, "China's renewable energy law and its impact on renewable power in China: progress, challenges and recommendations for improving implementation," Energy Policy, vol. 51, pp. 89-109, 2012.

[18] P. Shugang, "China’s legal system for water management: basic challenges and policy recommendations," International Journal of Water Resources Development, vol. 26, no. 1, pp. 3-22, 2010.

[19] A. Mars and M. S. Gouider, "Big data analysis to features opinions extraction of customer," Procedia Computer Science, vol. 112, pp. 906-916, 2017.

[20] C. Tietje and M. Lehmann, "The role and prospects of international law in financial regulation and supervision," Journal of International Economic Law, vol. 13, no. 3, pp. 663-682, 2010. 
[21] G. Nivetha and K. Venkatalakshmi, "Hybrid outlier detection (HOD) method in sensor data for human activity classification," Intelligent Data Analysis, vol. 22, no. 2, pp. 245-260, 2018.

[22] W. Dai, "Development and supervision of robo-advisors under digital financial inclusion in complex systems," Complexity, vol. 2021, Article ID 6666089, 12 pages, 2021.

[23] X. Wang, B. Zhang, F. Zhang, G. Teng, Z. Sun, and J. Wei, "Toward robust activity recognition: hierarchical classifier based on Gaussian process," Intelligent Data Analysis, vol. 20, no. 3, pp. 701-717, 2016.

[24] N. Moloney, "EU financial market regulation after the global financial crisis: "More Europe" or more risks?," Common Market Law Review, vol. 47, no. 5, 2010. 